Marquette University

e-Publications@Marquette

Physics Faculty Research and Publications

Physics, Department of

$1-1-2006$

Simulated Domain Wall Dynamics in Magnetic Nanowires

Andrew Kunz

Marquette University, andrew.kunz@marquette.edu

Published version. Journal of Applied Physics, Vol. 99, No. 8 (2006). DOI. (c) 2006 American Institute of Physics. Used with permission. 


\title{
Simulated domain wall dynamics in magnetic nanowires
}

\author{
Andrew Kunz ${ }^{\mathrm{a})}$ \\ Department of Physics, Marquette University, Milwaukee, Wisconsin 53233
}

(Presented on 3 November 2005; published online 28 April 2006)

\begin{abstract}
The simulated domain wall dynamics in rectangular $10 \mathrm{~nm}$ thick, $2000 \mathrm{~nm}$ long Permalloy wires of varying width is presented. In the absence of an applied field the static domain wall length is found to be linearly dependent to the width of the nanowire. As magnetic fields of increasing strength are applied along the wire's long axis, the domain wall motion changes from a uniform reversal to a steplike reversal. The onset of the stepping motion leads to a decrease in the domain wall speed. By continuing to increase the field it is possible to decrease the time between steps increasing the domain wall speed. The critical field associated with the crossover from uniform to nonuniform reversal decreases as the wire width increases. () 2006 American Institute of Physics.
\end{abstract} [DOI: $10.1063 / 1.2176890]$

\section{INTRODUCTION}

Scientifically magnetic nanowires offer the opportunity to study magnetic phenomena between atomic and bulk limits. ${ }^{1}$ Technologically the nanowires show promise for applications in high-density recording ${ }^{2}$ and spintronic sensing devices. ${ }^{3}$ To be useful in devices it is necessary to both understand the domain wall motion and control the switching behavior of the magnetic moments inside the wire. ${ }^{4}$ Electron spin currents have been shown to excite domain wall motion in nanowires, leading to some nonlinear effects ${ }^{5}$ and interesting observed domain wall velocity characteristics. ${ }^{6}$ In this paper micromagnetic simulations of the domain wall dynamics of a simplified model in which the walls are moved with external magnetic fields are presented. It is found that the presence of an external field can lead to irregular domain wall motion and an increase in the overall switching time.

\section{SIMULATION DETAILS}

The nanowires are simulated by numerically integrating the three-dimensional Landau-Lifshitz equation with Gilbert damping (LLG). ${ }^{7}$ The LLG equation describes the precessional motion of individual magnetic moments due to all internal and external fields. In this paper the rectangular nanowires are $2000 \mathrm{~nm}$ long and $10 \mathrm{~nm}$ thick, with widths that vary from 50 to $200 \mathrm{~nm}$. The simulated material was Permalloy with a saturation magnetization of $800 \mathrm{emu} / \mathrm{cm}^{3}$, an exchange constant of $1.3 \times 10^{-6} \mathrm{erg} / \mathrm{cm}$, no crystalline anisotropy, and a Gilbert damping parameter of $0.08 .^{8}$ The nanowire is discretized into identical cubic blocks of uniform magnetization, with dimensions of 2.5 and $5.0 \mathrm{~nm}$ on edge. The discretization size had no noticeable effect on the results. The large number of elements allows for a detailed view of the domain wall structure. Subpicosecond time steps were used to simulate the dynamics of the domain wall under the influence of externally applied magnetic field.

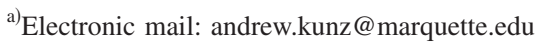

\section{RESULTS}

The static domain structure was determined for a domain wall located at the center of the wire. A sharp head to head, or transverse, wall was placed into the center of the wire. The magnetic moments were allowed to relax in zero external field to a stable configuration. The inset of Fig. 1 shows the top view of the magnetic structure for a transverse wall, where every other magnetic moment in both in-plane directions is represented by an arrow and by the gray scale. The domain wall is characterized by a vortex with an axis perpendicular to the length of the nanowire. The energy is minimized when the magnetic moments on either side of the wall point out of the material. Domain walls tend to stabilize when the moments at the ends of the walls point perpendicularly out of the material. ${ }^{9}$ As the width of the nanowire is increased from 50 to $200 \mathrm{~nm}$ the stable length of the domain wall grows proportionally as shown in Fig. 1. The domain wall length is typically found to be about $60 \%$ of the width of the wire.

An external field is applied to the equilibrium structure,

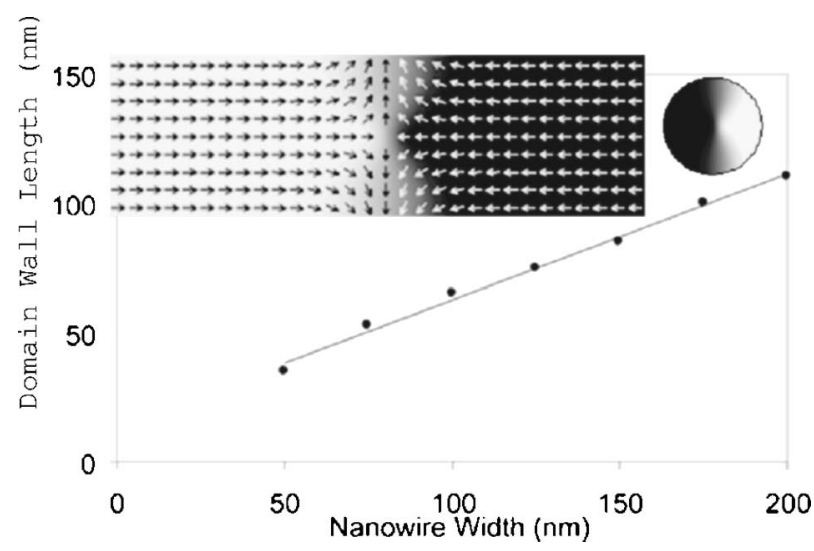

FIG. 1. Plot of the simulated domain wall width as a function of wire width in zero applied field showing a linear relationship. The inset figure is a top view of a $300 \mathrm{~nm}$ long segment containing the static magnetic structure of the transverse wall in a $100 \mathrm{~nm}$ wide wire where every second moment in both in-plane directions is shown. The circular inset indicates the relation between the gray scale and the direction of the in-plane component of magnetization. 


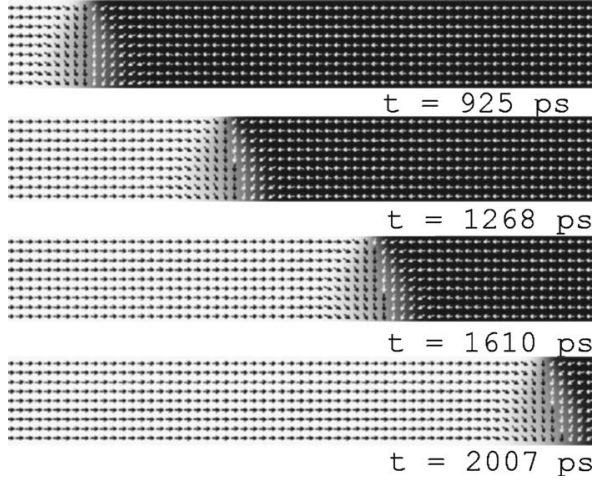

FIG. 2. Domain wall evolution in a $100 \mathrm{~nm}$ wide wire in the presence of a 100 Oe field to the right. The domain wall travels uniformly down the wire in the direction of the field. A third of the wire's total length is shown.

directly along the long $(x)$ axis of the wire, moving the wall down the wire's length. Independent of the wire's width, applied fields under 100 Oe set the domain wall into steadystate propagation down the wire in the direction of the applied field. Figure 2 shows a time lapse sequence of the motion of the domain wall in a $100 \mathrm{~nm}$ wide wire with an applied field of 100 Oe. Figure 3 is a plot of $M_{x} / M_{s}$ as a function of time corresponding to the same wire and field. The linear relationship further confirms the steady-state wall motion down the length of the wire.

Figure 3 also shows the change in $M_{x} / M_{s}$ as a function of time in the same $100 \mathrm{~nm}$ wide wire for an applied field of 140 Oe. The motion is quite different and can be characterized by the onset of plateaus where $M_{x} / M_{s}$ is constant in time. Figure 4 is a time lapse representation of the domain wall motion from about 2000 to 4000 ps, corresponding to the motion between subsequent plateaus in Fig. 3. In this representation the plateaus correspond to metastable states where the domain wall structure is similar to the zero applied field equilibrium structure shown in Fig. 1 ( $t=1984$ ps). Energetically speaking this period of time corresponds to peri-

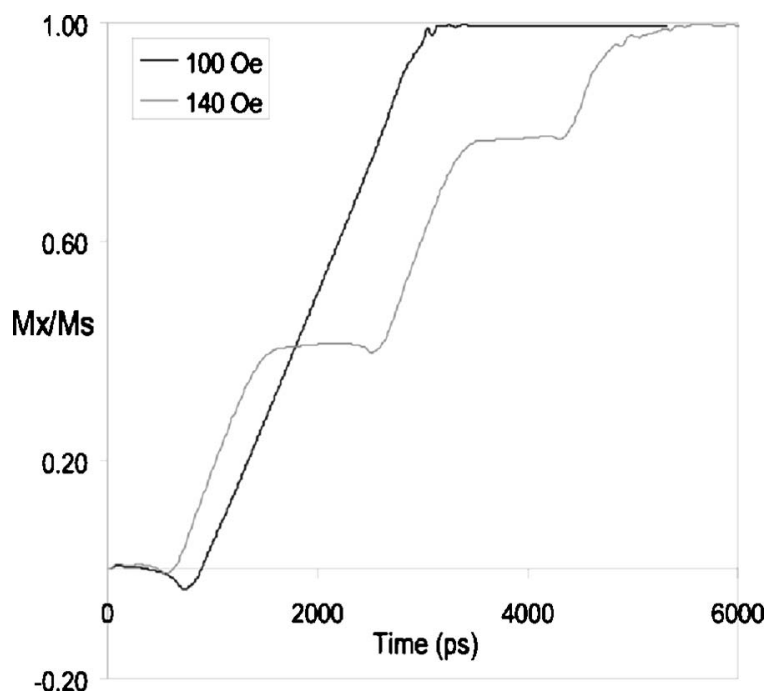

FIG. 3. $M_{x} / M_{s}$ as a function of time corresponding to the motion of domain walls in a $100 \mathrm{~nm}$ wide wire for 100 and 140 Oe fields. The $x$ direction is along the long axis of the wire. The plateaus are a result of induced dynamics within the domain wall region by the increasing magnetic field.

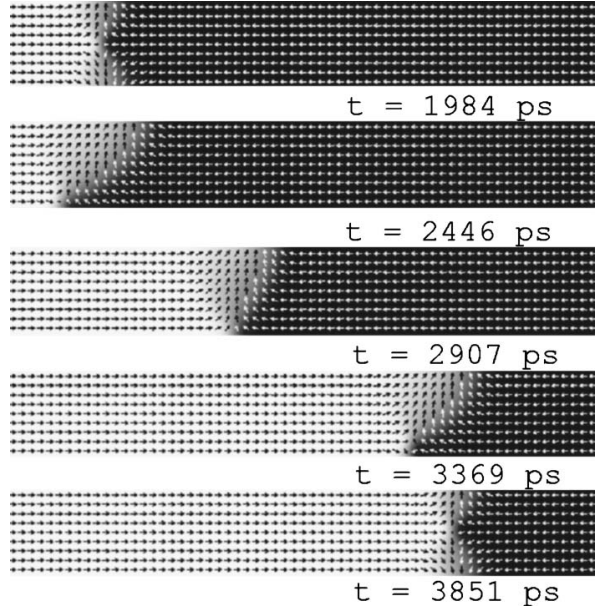

FIG. 4. Domain wall evolution in a $100 \mathrm{~nm}$ wide wire in the presence of a 140 Oe field to the right. The domain wall ratchets down the wire in a nonuniform manner with intervals of uniform motion and intervals of dynamics only within the wall. A third of the wires total length is shown.

ods of increased exchange energy which needs to be overcome by the external field. The exchange energy is relatively constant as the central wall vortex moves toward the wire's edge aligning the moments within the wall $(t=2446 \mathrm{ps})$. As the vortex reaches the edge there is a large drop in the exchange energy, and the wall begins to travel down the wire $(t=2907 \mathrm{ps})$. The wall then travels uniformly down the wire but with one end of the wall leading the other $(t=3369 \mathrm{ps})$. This expanding of the domain wall increases the wall energy. When the leading edge of the domain wall gets too far ahead, a vortex is nucleated at the trailing edge and another plateau is reached $(t=3851 \mathrm{ps})$. The process continues in this manner, with the domain wall traveling in a ratcheting manner down the wire. It is noted that the simulated nanowires have perfectly smooth edges so there are no inherent pinning sites on the wire, unlike what might be expected to happen experimentally.

To quantify the results it is helpful to look at the speed at

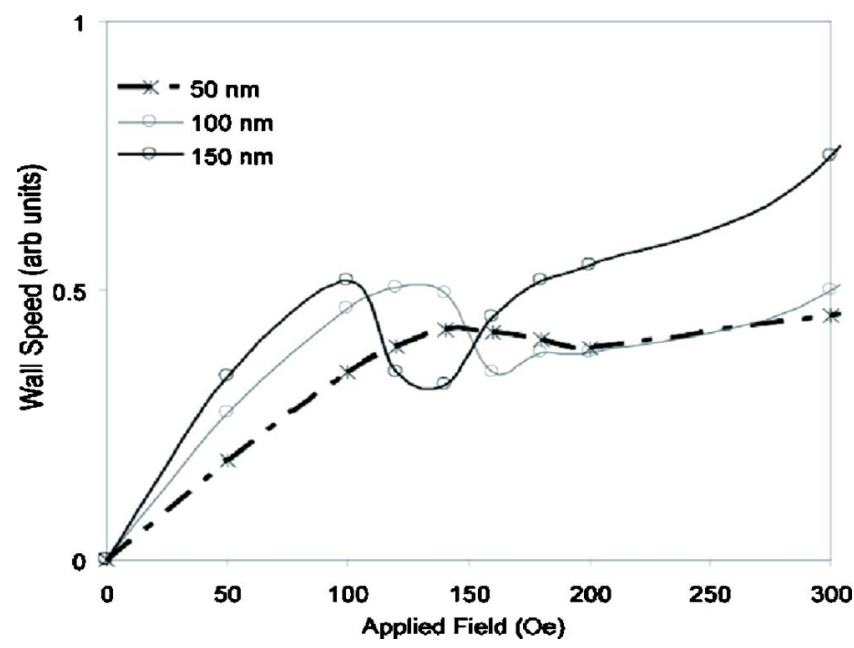

FIG. 5. (Color online) Plot of the domain wall speed as a function of applied field for three different wire widths. The initial linear increase in speed corresponds to uniform motion along the wire. The peak and subsequent dip appear when the walls begin ratcheting down the wire. The speed increases again when the applied field is strong enough to quickly unpin the walls. 
which the domain wall moves down the wire. The results are summarized in Fig. 5, the domain wall speed as a function of applied field for three different wire widths. Figure 5 shows that the domain wall speed increases as the magnetic field increases as long as the applied field is less than 100 Oe for all wire widths. As the external field is further increased, a critical field is reached at which point the motion ceases to be uniform. The nonuniformity in the motion causes the domain wall speed to decrease. Increasing the field further causes the speed to decrease even more before it begins to speed up again for still larger fields. This increase in speed still involves ratcheting behavior, but the length of time spent in the metastable plateaus decreases due to the increased Zeeman energy.

\section{CONCLUSIONS}

A detailed series of three-dimensional LLG simulations has been carried out to study the evolution of domain walls within a magnetic nanowire under the influence of an applied field. As the field strength is increased the domain wall motion undergoes a transition from uniform to nonuniform characterized by a ratcheting of the domain wall down the length of the wire. Calculations of the domain wall speed over the range of fields studied show similar behavior to that observed in spin-torque experiments, with an initial increase in domain wall speed followed by a decrease. This decrease in speed is also characterized by the onset of nonuniform wall motion.

\section{ACKNOWLEDGMENTS}

The author would like to thank the Helen Klingler College of Arts and Sciences and the Marquette University Summer Faculty Fellowship Program for supporting this work.

${ }^{1}$ J. Shi, S. Gider, K. Babcock, and D. D. Awschalom, Science 271, 937 (1996).

${ }^{2}$ C. A. Ross et al., Phys. Rev. B 62, 14252 (2000).

${ }^{3}$ G. Prinz and K. Hathaway, Phys. Today 48(4), 24 (1995).

${ }^{4}$ R. Wieser, U. Nowak, and K. D. Usadel, Phys. Rev. B 69, 064401 (2004). ${ }^{5}$ P.-B. He and W. M. Liu, Phys. Rev. B 72, 064410 (2005).

${ }^{6}$ A. Yamaguchi, T. Ono, S. Nasu, K. Miyake, K. Mibu, and T. Shinjo, Phys. Rev. Lett. 92, 077205 (2004).

${ }^{7}$ LLG Inc., Tempe, AZ 85282, USA.

${ }^{8}$ Standard Problem No. 4, http://www.ctcms.nist.gov/rdm/mumag.org.html ${ }^{9}$ G. D. Skidmore, A. Kunz, C. E. Campbell, and E. D. Dahlberg, Phys. Rev. B 70, 012410 (2004). 